\title{
EL CAMINO HACIA LA IGUALDAD DE GÉNERO EN LA EDUCACIÓN ESCOLAR. EL CASO DE EL SALVADOR (2016-2020)
}

\section{THE ROAD TO GENDER EQUALITY IN SCHOOL EDUCATION. THE CASE OF EL SALVADOR (2016-2020)}

\author{
Carlos Martínez Herrer
}

\begin{abstract}
The path taken by Salvadoran society to achieve gender equality in non-university education is undeniable. The implementation of numerous companies, the technical assistance of many countries, the collaboration of international institutions, and their financial support evidence, baking data extracted from the official censuses, that El Salvador is at the forefront of Hispanic Americans countries in equality policies. This article analyzes the data published by the entities working on gender equality and oficial agencies in El Salvador, for a present portrait and immediate future of gender equality policies in education. The "Human Capital" project 2016-2020, implemented by the Ministry of Education, and financial and technical assistance of the Millennium Challenge Corporation (MCC) will be further analyzed.
\end{abstract}

Key words: El Salvador, MINED, gender equality, education policy, equality policies

\section{RESUMEN}

El camino emprendido por la sociedad salvadoreña para conseguir la igualdad de género en la educación escolar, así como en la orientación académica y vocacional del alumnado, es innegable. La implementación de numerosas leyes, la ayuda técnica de numerosos organismos, la colaboración de instituciones internacionales, y el apoyo financiero de las mismas permiten decir, con apoyo en los datos extraídos de los censos oficiales, que El Salvador está en la vanguardia de los países hispanoamericanos en las políticas de igualdad de género en el ámbito de la educación. El presente artículo analiza los datos publicados por las entidades que trabajan en la igualdad de género en el Salvador, para hacer un retrato presente y de futuro inmediato de las políticas de igualdad de género en materia educativa. Se analizará más detenidamente el proyecto "Capital humano" 2016-2020, implementado por el Ministerio de Educación con la asistencia técnica y financiera de la Corporación Reto del Milenio (MCC) y se presentará a modo de conclusiones los aspectos que dificultan la puesta en práctica de la política de igualdad de género en la educación escolar.

Palabras clave: El Salvador, MINED, igualdad de género, política educativa, políticas de igualdad.

Fecha de recepción: 31 de julio de 2018.

Fecha de aceptación: 18 de enero de 2019 


\section{INTRODUCCIÓN}

Hace ocho años, el 15 de abril de 2010, AECID el Salvador, la entidad de Cooperación al Desarrollo del Ministerio de Asuntos Exteriores de España, presentaba a la opinión pública salvadoreña el informe elaborado por el Consejo por la Igualdad y la Equidad (CIE) "Paso a paso hacia la igualdad de género en El Salvador" (CIE, 2009). El informe recomendaba al Gobierno de la República fortalecer el ISDEMU -el organismo rector de las políticas de igualdad de género en el país-, dotarlo del financiamiento necesario e impulsar la igualdad a través de la participación de la sociedad civil. Tres pasos para la acción con los que se perseguía apoyar al desarrollo humano, puesto que las desigualdades de género estaban en la base de los problemas más graves que sufría y sufre el país.

El informe fue entregado a la secretaria de Inclusión Social de entonces y ahora, Vanda Pignato, y era fruto del trabajo llevado a cabo en el seno del CIE, un espacio de reflexión, diálogo y seguimiento del avance de la igualdad de género en el país, creado en septiembre de 2009 y que cuenta con el apoyo del Programa de Las Naciones Unidas para el Desarrollo (PNUD), el Fondo de Población de las Naciones Unidas (UNFPA), la Agencia Española de Cooperación Internacional para el Desarrollo (AECID) y la Agencia Andaluza de Cooperación Internacional para el Desarrollo (AACID-Junta de Andalucía).

Las recomendaciones del CIE partían de un análisis sobre la igualdad de género en El Salvador realizado en cuatro áreas prioritarias: la participación política, la violencia de género, la pobreza y la salud.

\subsection{PARTICIPACIÓN POLÍTICA}

El diagnóstico señalaba que la reducida participación de las mujeres en los espacios de decisión política se producía en un contexto normativo y de políticas que, en principio, debía ser favorable a su incremento. Además, su presencia era minoritaria cuando ello dependía de los procesos de decisión política -el Ejecutivo de entonces contaba con un $15 \%$ de mujeres y la Asamblea Legislativa con un 19\%- y aumentaba cuando la selección dependía de la promoción profesional en el poder judicial, las juezas representaban el $44 \%$ de la carrera judicial en 2010-.

La brecha de la discriminación también se trasladaba al ámbito municipal, donde sólo 29 municipios de los 262 existentes en el país estaban regidos por mujeres. Para enfrentar esta situación, el CIE apostaban por el sistema de cuotas en la normativa electoral, junto con la capacitación de las mujeres en el desempeño de cargos de responsabilidad, así como por la creación de mecanismos para armonizar las responsabilidades laborales y familiares de las mujeres, las políticas de conciliación.

\subsection{VIOLENCIA DE GÉNERO}

El informe también reflexionaba sobre la violencia de género. Aunque los hombres eran las víctimas del $90 \%$ de los homicidios en el país, la tasa femenina había ido incrementándose, siendo la violencia de género la causa del $50 \%$ de las muertes violentas de la mujer. Frente a la alarmante situación de violencia, el CIE sugería incluirla como uno de los tipos de violencia a combatir dentro de los planes del Ejecutivo, además de la creación de una normativa integral, acompañada de estrategias de sensibilización. 


\subsection{POBREZA}

En El Salvador, la pobreza, tal y como recogía el documento, es una situación que compete tanto a hombres como a mujeres. No obstante, al examinar las condiciones del empleo se observaba una brecha salarial. La remuneración obtenida por las mujeres en 2007 era un $86.1 \%$ de la que obtenían los hombres. El resto de datos resumían "lugares comunes": que las mujeres trabajan más, tienen peores condiciones de empleo y menor salario promedio, (pero recibían remesas en mayor cantidad que los hombres, factor que debemos atribuir a circunstancias familiares). En esta área el CIE recomendaba generar una política integral para el combate de la pobreza y la exclusión, pero incorporando criterios de género, poniendo el acento en la educación y la autonomía económica en el mercado de trabajo.

\subsection{SALUD}

En el ámbito de la salud, el informe señalaba que la situación en el país era "todavía precaria”, tanto para mujeres como para hombres. Si bien afectaban a las mujeres algunos de los grandes retos para el desarrollo de El Salvador, como son los embarazos adolescentes: las mujeres embarazadas entre los 10 y 19 años representaban un 31\%, con todo lo que ello supone de riesgo para su salud, además de limitaciones académicas y dificultades de autonomía económica.

Las medidas sugeridas por el CIE en este sector pasaban por una reforma integral del sistema de salud que incorporara el enfoque de género, no sólo en el sentido de tratar la salud de la mujer superando la perspectiva materno infantil, sino también identificando los roles de género que conducen a los hombres a presentar comportamientos de riesgo.

En esta iniciativa, como en el resto de las que se apoyaban en materia de género en El Salvador, la AECID iba de la mano con sus principales socios multilaterales y la Agencia Andaluza, para conseguir una mayor eficacia de la Ayuda Oficial al Desarrollo (AOD) y para cumplir con sus compromisos de alineamiento, armonización y apropiación.

Sobre este punto de partida, la meta general segunda del Horizonte Educativo 2021 pretende lograr la igualdad educativa y superar toda forma de discriminación en la educación, planteándose en la meta específica tercera "prestar apoyo especial a las minorías étnicas, poblaciones originarias y afrodescendientes, a las alumnas y al alumnado que vive en zonas urbanas marginales y en zonas rurales, para lograr la igualdad en la educación". Ciertamente, es una meta ambiciosa, que plantea "desigualdades intercurrentes": las basadas en raza, origen, sexo y la clásica distinción entre ámbito urbano y ámbito rural. Todos estos factores están presentes en el camino hacia la equidad de género y serán objeto de análisis en los párrafos siguientes. Como corresponde a una meta ambiciosa, el indicador de logro para el año 2021 es también ambicioso: "El porcentaje de niños de minorías étnicas, poblaciones originarias y afrodescendientes, residente en zonas urbanas marginales y zonas rurales, y de género femenino, es al menos igual a la media del alumnado escolarizado en la educación inicial, primaria y secundaria básica”.

\section{ANÁLISIS ACTUAL DE SITUACIÓN SOCIAL Y DE GÉNERO EN EL SALVADOR.}




\subsection{INTRODUCCIÓN.}

La categoría género determina el sistema de simbolización y valoración de las personas, entrelaza y potencia constituyentes de jerarquización y diferenciación basados en relaciones de autoridad, de pertenencia, que jerarquiza a ciertos colectivos a partir de juicios muchas veces no explicitados. Los que generalmente están internalizados en las actuaciones sociales, son actos de exclusión no necesariamente de forma reflexiva, y que muchos autores circunscriben a la subcategoría no exclusiva de micromachismos (Ruiz-Jarabo et Blanco, 2005), cuyas manifestaciones son sutiles y cambian en su forma de expresión. La presencia de estas relaciones de poder provoca el surgimiento de inequidades de género que se enuncian a través del acceso y control no equitativo sobre los recursos, entendidos como los bienes o servicios a disposición de las personas. Dentro de estos recursos se pueden señalar la información técnica y conocimientos, acceso a recursos económicos y financieros, acceso a los procesos de toma de decisión, uso de nuevas tecnologías, la protección frente a la violencia y el acoso; el acceso a los ámbitos públicos, la apreciación del trabajo, el reconocimiento de insuficiencias e intereses y el pleno goce de los derechos labores y ciudadanos. Estas desigualdades son las llamadas brechas de género (OCDE, 2014) y están sustentadas con datos cuantitativos, que permiten identificar las diferencias entre mujeres y hombres al acceso de los recursos generados en los procesos de desarrollo. La lógica de la inequidad de género tiene su fundamento en la división sexual del trabajo (, en imágenes o estereotipos que han asociado el sexo de una persona a sus ámbitos de actuación, así como a su capacidad para realizar una ocupación, confiriendo la esfera pública a los hombres y la esfera privada a las mujeres, ubicándolas en una situación de desventaja con relación a los hombres. La esfera pública representa el quehacer productivo y remunerado de los hombres, mientras la esfera privada representa el trabajo dentro de casa, privado, doméstico, el cual ha sido asignado mayoritariamente a las mujeres y sobre el cual no hay remuneración monetaria. En este contexto, la lógica de las inequidades de género se refleja de forma diferente para cada sector productivo de un país, aunque es un patrón constante la infrarrepresentación de la mujer en los sectores productivos asociados a la producción científica, la producción técnica, la producción económicamente más rentable y los puestos asociados a la toma de decisión e influencia social.

\subsection{ANÁLISIS CUANTITATIVO. ALGUNOS DATOS ESTADÍSTICOS.}

En El Salvador se han alcanzado progresos importantes en diferentes ámbitos de la vida social, económica y política de las mujeres. Esto es el corolario, en gran medida, de los logros en los procesos de transversalización del principio de igualdad y la institucionalización de políticas oficiales a favor de las mujeres, lo cual ha permitido el desarrollo, aplicación y seguimiento de marcos legales en materia de inclusión, igualdad de oportunidades y equidad de género. Una muestra de estos avances es la creación de Unidades de Género en instituciones públicas y Unidades de la Mujer en Gobiernos Municipales; políticas internas de género en los diversos Ministerios de la República, de acuerdo a lo que establece la Ley de Igualdad, Equidad y Erradicación de la Discriminación contra las Mujeres, y políticas institucionales de género. (ISDEMU, 2013). La Unidad de Género del Ministerio de Educación (MINED) fue creada por Acuerdo no 15-1269 del MINED, en agosto de 2016. La Encuesta de Hogares y Propósitos Múltiples (EHPM) de 2013 refleja que la población total del estado para ese año era de 6.29 millones de individuos, de las cuales el $56.2 \%$ correspondía a menores de 30 años (jóvenes), mientras que la población de 60 años y más (ancianos) representaban el 11.0\%. Esto revela que la población salvadoreña es bastante joven, lo que facilita la renovación generacional en los ámbitos productivos y de la sociedad en general. Al diferenciar la población por sexo, se observa que las mujeres representaban el 52.6\% de la población total del país y los hombres el $47.4 \%$, obteniéndose un índice de masculinidad de 0.90. En la zona urbana este índice es de 0.86 y en la rural de 0.96 
(DIGESTYC, 2014). De acuerdo con el ISDEMU, si bien existen progresos orientados a la disminución de las inequidades de género, todavía persisten brechas significativas entre mujeres y hombres.

Desde la orientación de los ejes temáticos de la Política Nacional de las Mujeres y del Plan Nacional de Igualdad y Equidad para las Mujeres Salvadoreñas, las principales desigualdades se detallan a continuación (ISDEMU, 2013):

\subsubsection{APORTACIÓN ECONÓMICA}

Las mujeres tienen menores oportunidades de trabajar de forma remunerada. Para el año 2013, la tasa de participación laboral específica fue de $49.3 \%$ para las mujeres y del $80.7 \%$ para los hombres; es decir, por cada 100 individuos en edad de trabajar, 49 mujeres estaban ocupadas u ofertando su fuerza de trabajo al mercado laboral, muy lejos de los hombres, quienes 81 de cada 100 se hallaban en esta misma situación.

El desempleo es casi el doble entre la población joven que en el agregado de la población trabajadora. La tasa de desempleo se sitúa en $9.9 \%$ para la población activa entre 16 y 29 años, que resulta superior a la nacional, ubicada en $6.0 \%$. A nivel global, el desempleo parece ser menor en las mujeres, aunque el dato en realidad encubre las dificultades de ingreso a las profesiones que requieren estudios universitarios por parte de las mujeres. Para el grupo de edad mencionado, el desempleo femenino se ubica muy cerca del masculino ( $11 \%$ hombres y $9.7 \%$ mujeres). (DIGESTYC 2013)

Segmentación laboral afecta a las mujeres. Esta se formula tanto en los empleos a nivel horizontal, donde en la práctica se evidencian "trabajos de hombres" y "trabajos de mujeres", trasunto de la teoría de las dos esferas, y que son en general una prolongación de sus actividades tradicionales (educación, salud, trabajo doméstico, comercio, servicios, trabajo sin remuneración, etc.) La tasa de participación laboral específica mide el grado de participación de la población en el mercado de trabajo y se obtiene del cociente de la Población Económicamente Activa (PEA) y la Población en Edad de Trabajar (PET) para cada sexo, como a nivel vertical (la mayoría en los puestos de decisión son ocupados por hombres), aunque la brecha educativa entre mujeres y hombres es bastante reducida. En cuanto a calidad y circunstancias del trabajo, los hombres acceden en mayor medida al mercado formal de trabajo, por lo que tienen mejores salarios y condiciones laborales, permanencia en el empleo y acceso a la protección social, mientras que las mujeres en su mayoría trabajan en el mercado informal, caracterizado por la prevalencia de condiciones de trabajo precarias, inestabilidad, salarios bajos, y reducido acceso a la protección social.

Tasa de mujeres que viven en pobreza es más alta que la de los hombres. Las mujeres en pobreza alcanzan un $34.8 \%$ y los hombres $29.6 \%$. La brecha entre hombres y mujeres en situación de pobreza extrema alcanza un $6 \%$ de diferencia y ubica a las mujeres en mayor precariedad (DIGESTYC, 2013).

En general las mujeres reciben menor retribución que los hombres. Para trabajos de igual responsabilidad se constata una brecha importante entre mujeres y hombres, ya que el salario medio de las mujeres equivale en promedio entre un 15 y $20 \%$ menos que el de los hombres.

\subsubsection{EDUCACIÓN}


Alfabetización: más mujeres analfabetas que hombres. La tasa promedio de analfabetismo para la población en general descendió del 28\% en 1992 al 13\% en 2011 (PNUD). No obstante, se mantiene una brecha de género que desfavorece mayoritariamente a las mujeres del área rural. La información desagregada de la EHPM para el año 2013 reflejaba que las tasas específicas de analfabetismo eran: a nivel nacional, para las mujeres el 13.7\%, y para los hombres el $9.6 \%$. En las áreas urbanas para las mujeres era del $9.5 \%$ y para los hombres de $5.3 \%$; en el mundo rural, para las mujeres era del $21.2 \%$ y para los hombres de 16.5\%. La información desagregada de la Encuesta de Hogares de Propósitos Múltiples (EHPM) para el año 2014 refleja que las tasas específicas de analfabetismo son: a nivel nacional, para las mujeres del $12.4 \%$, y para los hombres del $9.0 \%$, lo que evidencia una brecha de 3.4 puntos porcentuales que aventaja a los hombres, En el área urbana para las mujeres es del $8.7 \%$ y para los hombres del $4.9 \%$; en lo rural, para las mujeres es del $19.2 \%$ y para los hombres del 15.8\%. Las diferencias de la tasa de analfabetismo se localizan en los rangos de edad de las personas mayores de 30, pero la comparación entre 2013 y 2014, últimos datos disponibles, refleja una evidente mejora en el ítem estadístico.

La tasa de asistencia escolar es menor para las mujeres: para el 2013, la EHPM señalaba que, a partir de la educación preescolar, la tasa de asistencia fue 31.1\% (del total de población de 4 años y más). La tasa de asistencia escolar específica de los hombres a nivel estatal era de 33.5\% y de las mujeres de $28.9 \%$. En el área urbana, la asistencia escolar específica de los hombres era de $34.7 \%$ y de las mujeres de $29.2 \%$; en el área rural, el $31.4 \%$ de los hombres asistían a un centro escolar; las mujeres lo hacían en 28.4\%. En términos de la población global, para el año 2014 la EHPM señala que la tasa de asistencia fue del 28.7\%, la misma que el año anterior (del total de población de 4 años y más). Al comparar por sexo, la tasa de asistencia escolar específica de los hombres a nivel nacional fue del $30.9 \%$ y de las mujeres del $26.6 \%$. En el área urbana, la asistencia escolar específica de los hombres es del $32.0 \%$ y de las mujeres del $27.1 \%$; en el área rural el $29.2 \%$ de los hombres asisten a un centro escolar; las mujeres lo hacen en un $25.8 \%$.

Aunque la brecha se ha reducido, el promedio de años de escolaridad de las mujeres es menor que el de los hombres: a nivel nacional, la escolaridad promedio por sexo es de 6.7 grados (cursos anuales) para los hombres y 6.5 para las mujeres datos señalados por la EHPM 2013. En el área urbana, la escolaridad promedio de los hombres es de 7.9 grados y de las mujeres de 7.5 ; en la zona rural, la de los hombres es de 4.9 grados y de las mujeres de 4.6 grados. Al desagregar la escolaridad promedio por departamento, los que reflejan más grados de escolaridad son San Salvador y La Libertad, (los departamentos con mayor riqueza), con 8.2 y 6.9 grados de escolaridad, respectivamente. La Unión y Morazán (los departamentos con menor riqueza) muestran los niveles más bajos de escolaridad: 4.7 y 5.0 grados, respectivamente.

El promedio de años de estudio de las mujeres es levemente menor que el de los hombres: a nivel nacional, la escolaridad promedio por sexo es de 6.9 grados para los hombres y de 6.7 para las mujeres, datos señalados por la EHPM 2015. En el área urbana, la escolaridad promedio de los hombres es de 8.1 grados y de las mujeres de 7.7; en la zona rural, la de los hombres es de 5.1 grados y de las mujeres de 4.9 grados.

Razones de inasistencia. En relación con las razones por las que no asisten a la escuela las niñas y los niños entre 4 a 17 años de edad, representando el porcentaje de mujeres sobre el total de mujeres que no asiste y de hombres sobre el porcentaje total que no asiste. Destacan razones diferenciadas por sexo que están asociadas a los patrones culturales de los roles asignados a cada sexo. En menor proporción se señala el embarazo y maternidad. 
Fracaso escolar de niñas, niños y jóvenes: las tasas de repetición en el periodo 2008 - 2010 son mayores en $1^{\circ}$ y $7^{\circ}$ grado, siendo estas mayores en los niños. Las tasas más altas de deserción se tienen en $9^{\circ}$ grado, seguido de $7^{\circ}$ y $1^{\circ}$ grado. Ni estudian ni trabajan (Jóvenes “nini”): El 12.2\% de adolescentes entre 14 y 17 años no estudia ni trabaja con derecho a remuneración, una situación que se agudizada en el área rural, donde el porcentaje aumenta a un $18.2 \%$, y a un $8.0 \%$ en lo urbano. A nivel nacional y por sexo, las adolescentes que no estudian ni trabajan representan un $17.8 \%$, frente al $6.7 \%$ de los adolescentes varones. En el área rural, el 29.4\% de las adolescentes estaría en esta situación, es decir, 1 de cada 4 adolescentes rurales no estudia ni trabaja por una remuneración. Ciertamente, no está considerado en este dato desagregado la economía informal o sumergida.

Fracaso escolar de niños y jóvenes con respecto a las niñas. Las tasas de repetición, de acuerdo con la EHMP (2015), son mayores en el nivel de educación primario a nivel nacional y la tasa es mayor en los niños en relación con las niñas en los niveles de escolaridad primarios, tercer ciclo y educación media (ver anexo 1). Al respecto existen dos situaciones que reflejan la complejidad de la situación. La primera es que, a pesar que las niñas se encuentran en menor riesgo de fracaso escolar, tienen un rendimiento académico ligeramente menor al de los niños en pruebas nacionales como la PAES, que se acentúa más en materias como matemáticas y ciencias (MINED, 2014).

Fuerte segregación de los estudios de educación media técnica por sexo y su consecuente segregación en el mercado de trabajo. Con relación a la educación media técnica observa brechas de género en las áreas formativas de las especialidades del bachillerato producto de una división tradicionalmente de los considerado "propio" de hombres y "propio" de mujeres. Si bien esta brecha afecta a ambos sexos, son precisamente las mujeres quienes se concentran en menos opciones y tienden a optar por estudios asociados mayoritariamente a aspectos sociales y relacionados con el cuidado (salud, educación, servicios) mientras que los jóvenes del sexo masculino acceden a más y diversas opciones asociadas mayoritariamente a los ámbitos técnicos. Esta segregación limita el potencial de las mujeres para acceder a una mayor variedad de empleos y emprendimientos técnicos y por ende de crecimiento de sus ingresos. El trasfondo de esta segmentación de la educación técnica tiene incidencia a futuro en el desarrollo de las mujeres, que al ubicarse en el mercado de trabajo, son afectadas, ya que los sectores de mayor participación laboral de mujeres se caracterizan por una menor remuneración y menor tecnificación en comparación al resto de actividades que tienen predominante participación de hombres. De igual forma afecta el desarrollo del mercado laboral, que podría beneficiarse con los aportes tanto de las mujeres como de los hombres. Esta tendencia pareciera repetirse en la formación profesional y la habilitación para el trabajo.

El acceso a la formación técnica es menor para las mujeres. Otro indicador importante se encuentra en el acceso a la formación técnica profesional, al respecto del total de la población que accede a esta modalidad educativa, se observan diferencias que desfavorecen a las mujeres: $52 \%$ de hombres y 48\% de mujeres (DIGESTYC, 2014).

Alto número de adolescentes embarazadas. Reto para la prevención y continuidad educativa de madres adolescentes. El Salvador ocupa uno de los primeros lugares de los países latinoamericanos con la mayor tasa de embarazos en adolescentes (algunos son producto de violencia sexual). En promedio cada día entre 65 a 70 adolescentes menores de 19 años de edad se convierten en madres. El informe de Situación de la Niñez y Adolescencia en El Salvador. El Salvador UNICEF (2014), señala que esta cifra ha venido aumentando, ya que en 2005 se contabilizaron 21,693 y en 2014 se reportaron 24,802 . Se carece de un fiel registro y de indicadores nacionales sobre las adolescentes madres que continúan sus estudios. La prevención del embarazo precoz, así como la continuidad 
educativa de las adolescentes embarazadas y jóvenes con hijos constituye uno de los principales desafíos para el sistema educativo.

Uniones matrimoniales y no matrimoniales de adolescentes. La EHPM para el año 2014, señala que, a nivel nacional, 23,573 NNA de 12 a 17 años, tiene o han tenido una relación matrimonial o no matrimonial, esto representa el $2.8 \%$ de la población en este rango de edad (se incluyen casada/o, acompañada/o y separadas/os). De este grupo, el 57.4\% se encuentran en el área rural y el $42.6 \%$ en el área urbana. Para atender esta problemática se requiere la coordinación entre las diferentes instituciones, como la escuela, los servicios de salud y el sistema de protección en la realización de acciones integradas y conjuntas para dar una mejor respuesta a este complejo problema y erradicar esta práctica que todavía es aceptada socialmente.

Más allá de las estadísticas, hace falta investigar a profundidad la situación de discriminación y violencia de género en el ámbito educativo. El estudio realizado por FOMILENIO/ MINED (2013) señala que en los centros escolares existen normas de género que socializan estereotipos sexuales y prácticas educativas que colocan a las niñas, adolescentes y mujeres en riesgo de vivir en condiciones de desigualdad y subordinación respecto a los hombres. Niñas y niños son educados de forma diferente, antagónica y excluyente basados en la creencia de lo considerado "propio" de la identidad femenina en contraposición a lo considerado "propio" de la identidad masculina, que presupone la superioridad del sexo masculino sobre el femenino.

Paridad en la tasa neta en primaria. En El Salvador, se observa en los últimos años, un acceso a la escuela más paritario entre niñas y niños. De acuerdo con la Encuesta Nacional de Salud (2014) el Índice de paridad de género tomando la tasa neta para la escuela primaria es 1.0, lo que indica que no existe mayor diferencia entre niñas y niños al nivel de educación primaria, dado que el porcentaje de niños y niñas en edad de entrar a la escuela primaria que ingresan al grado 1 (tasa neta de admisión), es de $96.2 \%$ para las niñas y $95.4 \%$ de los niños.

Paridad en la tasa neta en Educación Secundaria. En cuanto a la asistencia a la educación secundaria que comprende tercer ciclo (séptimo a noveno grado) y educación media (primero a tercer año de bachillerato), los resultados indican una tasa neta de asistencia ajustada del $62.4 \%$ para los niños y del 64.1\% para las niñas. El Índice de paridad de género reportado para la escuela secundaria es de 1.03 , este indicador refleja un aumento ligeramente mayor a favor de las niñas

Entre los años 2000 y 2014, se ha producido un incremento de la tasa neta tanto para tercer ciclo como para bachillerato. En cuanto al Tercer Ciclo $\left(7^{\circ}\right.$ a $\left.9^{\circ}\right)$ la tasa se ha incrementado en 17.9 puntos porcentuales y para el nivel bachillerato ha aumentado del $27.2 \%$ en el 2000 a $37.9 \%$ en 2014, no obstante, aún persisten desafíos de cobertura educativa.

En cuanto a la posible identificación de brechas de género, los datos desagregados por sexo indican una tendencia que favorece a la población femenina y coloca en desventaja a la población masculina. Al respecto, la tasa neta de asistencia escolar para el tercer ciclo muestra una diferencia de 6.7 puntos porcentuales entre mujeres y hombres: para el sexo femenino es de 67.7 y para el sexo masculino de 61.0. En el nivel de educación media la brecha es de 4.6 puntos porcentuales a favor de la población del sexo femenino: 40.2 para mujeres y 35.6 para hombres. Aquí se hace uso de las tasas netas debido a que las tasas brutas de asistencia proporcionan una descripción errónea del Índice de Paridad de Género, principalmente porque la población estudiantil que ha superado la edad para asistir a la escuela primaria suelen ser niños. Si el indicador se encuentra en 1 se interpreta como paridad pero si sobre pasa dicho valor, este representa una inclinación a favor de las niñas (ENS, 2014). Es importante agregar que no existen estudios sobre las razones de tasas 
más equivalentes entre sexos. Más adelante se identifican diferencias de género que se basan en datos del total de la población femenina y masculina, a excepción de los datos anteriores que concentran el análisis en el grupo de edad entre los 7 y 18 años.

\subsubsection{PARTICIPACIÓN POLÍTICA Y CIUDADANA}

La participación política pública de las mujeres refleja avances significativos en los últimos años; sin embargo, las mujeres aún no logran participar en condiciones de igualdad con los hombres para ocupar cargos de representación paritaria en los tres órganos del Estado.

En el gabinete de Gobierno, la participación de las mujeres representa un 33\% con tres Ministras, cinco Viceministras, dos Secretarias de la Presidencia y siete Presidentas o Directoras de instituciones autónomas.

En la Asamblea Legislativa, 27 mujeres son diputadas, lo que representa el 33\% del total, y cinco de las personas que integran la Junta Directiva de la Asamblea Legislativa son mujeres, lo que representa el 37.5\%. Además, la presidenta de la Asamblea Legislativa es una mujer, la segunda en ocupar ese cargo en la historia.

En relación a la participación de las mujeres en los gobiernos locales, para el período 2012-2015, únicamente 28 mujeres fueron electas alcaldesas, lo que representa el 10.7\% de los y las titulares de los gobiernos municipales. El 18.7\% son síndicas (portavoces de grupos municipales); el 26.3\% son regidoras propietarias y el 31.5\% regidoras suplentes. En conjunto, en los Concejos Municipales el 26.1\% son mujeres. Para el período 2015-2018, únicamente 27 mujeres son Alcaldesas, lo que representa el 10.3\% de los y las titulares de los gobiernos municipales. El 24.4\% son Síndicas; el $26.3 \%$ son Regidoras Propietarias, el 28.7\% Regidoras Suplentes. En conjunto, en los Concejos Municipales el $42.1 \%$ son mujeres.

\subsubsection{VIOLENCIA DE GÉNERO}

La violencia contras las niñas y las adolescentes tiene en El Salvador proporciones preocupantes. Entre enero 2012 y junio 2014, una de cada cuatro mujeres asesinadas era menor de 19 años de edad. En el mismo periodo fueron denunciados más de 7.700 casos de agresión sexual a mujeres, de los cuales, el 66\% de las víctimas tenían entre 10 y 19 años. El gran problema a considerar en esta estadística, ciertamente, es la violencia oculta o no denunciada, un dato que puede representar más de la mitad de la violencia denunciada.

El ISNA (Instituto Salvadoreño de Desarrollo Integral para la Niñez y Adolescencia) informó que del total de casos de niñas y adolescentes víctimas de abuso sexual atendidas entre 2008 y 2010, al menos el 10\% tuvo como consecuencia un embarazo (UNICEF, 2014). Es importante resaltar, como se indicó en el párrafo anterior, que los datos se refieren exclusivamente a casos denunciados, que, como está mundialmente demostrado, son representativos con respecto al total de casos de violencia sexual que ocurren.

Según el Banco Mundial (2012), más de la mitad de los embarazos en adolescentes son consecuencia de una violación. Está comprobado además que cuanto más joven es la niña embarazada, mucho mayor es la probabilidad de que el embarazo sea consecuencia de una violación. Cabe resaltar que de acuerdo al código penal de El Salvador, la edad mínima de consentimiento es 15 años, por lo que cualquier embarazo de una menor de 15 años es resultado de una violación. 
Según el Observatorio de Seguridad Ciudadana (OEA, 2016) hubo un acumulado de 4.973 asesinatos de mujeres entre 1999 y 2016. Aunque entre el 2012 y el 2014 se vio una reducción de feminicidios (de 630 feminicidios en el 2011 a 292 en 2014), en el 2015 hubo un aumento de $61.47 \%$, con un total de 475 . Entre los contextos de feminicidios más comunes están feminicidios por violencia doméstica (por la pareja o expareja), asesinatos por pandilleros y/o "maras" por negarse a tener algún acceso carnal o vínculo afectivo con ellos, crímenes que son usados para marcar territorios o causar terror entre la población, y homicidios por desconocidos o conocidos para causar daño a parientes.

Existen avances en la legislación al contar con la Ley Especial Integral para una Vida Libre de Violencia para las Mujeres (2012), en tanto que han orientado la actuación de los poderes públicos para erradicar la violencia contra las mujeres y establecer mecanismos institucionales para acercar los servicios públicos de prevención, atención integral y protección.

Para la consolidación de estos avances es necesario reconocer que las mujeres y los hombres viven y ejercen sus derechos de manera diferenciada, y que los marcos legales y las prácticas jurídicas presentan profundas características sexistas en su interpretación y en su aplicación.

\subsubsection{INSEGURIDAD CIUDADANA.}

A pesar de los esfuerzos del Gobierno de la República, la violencia continúa siendo uno de los principales problemas del país, según lo indica el estudio presentado por la fundación Dr. Guillermo Manuel Ungo con datos oficiales del Instituto Nacional de Medicina Legal (Fundaungo, 2016). Para el periodo 2009 al 2014 se presentaron los siguientes resultados: en cuanto al número de homicidios, durante 2009 ocurrieron 4,382; en 2010, 4,004; y en 2011, 4,371. A partir de 2012 se evidencia una disminución importante, llegando a niveles de 2,594 homicidios en 2012; y, 2,499, en 2013. En 2014, se incrementó a 3,912, y de enero a diciembre de 2015 ocurrieron 6,640 homicidios.

Dentro del informe anual 2014 de Trata de Personas del Departamento de Estado de los EEUU, El Salvador se ubica en entre los países que todavía no cumplen completamente con los estándares mínimos para la eliminación de la trata, pero cuyos gobiernos están haciendo esfuerzos hacia esa meta. Aunque el gobierno aprobó la Ley Especial contra la Trata de Personas en el 2014, y creó el Consejo Nacional Contra la Trata de Personas, las definiciones son inconsistentes con las leyes internacionales, pues dejan sin protección a ciertos grupos, los servicios para víctimas son inadecuados y las investigaciones y enjuiciamientos no son llevados a cabo. La trata es un crimen muy difícil de detectar o denunciar y, por lo tanto, las estadísticas no son adecuadas para evaluar la magnitud del problema. De acuerdo con la Policía Nacional Civil (PNC), de 2013 a 2015 un total de 191 casos fueron denunciados, de los cuales 73\% fueron mujeres, 7\% fueron hombres y el restante 20\% no tiene sexo registrado. El $80 \%$ de estas víctimas de trata eran de El Salvador, mientras que el 20\% eran de otras nacionalidades, predominando las centroamericanas: Honduras, Nicaragua, Guatemala y Costa Rica, entre otras.

\section{EL PROYECTO “CAPITAL HUMANO" EN MATERIA DE EDUCACIÓN DEL GOBIERNO DE EL SALVADOR.}

\subsection{MARCO LEGAL E INSTITUCIONAL}


Sin duda, el proyecto más importante en materia de educación, en el periodo 2016-2020, del Gobierno de El Salvador es el denominado "Capital Humano", proyecto financiado por el Gobierno USA a través de la Corporación Reto del Milenio (MCC, en sus siglas en inglés).

Este proyecto, con una asignación presupuestaria de 115,7 millones de dólares, tiene como meta mejorar la calidad de la educación y alinearla mejor con las destrezas demandadas por los empleadores en el comercio internacional de bienes y servicios, para incrementar la productividad de la mano de obra de las trabajadoras y los trabajadores salvadoreños del futuro.

El Ministerio de Educación (MINED) es el ente rector de la educación y el órgano político que desarrolla e implementa las normas de equidad de género en educación, y también el proyecto de "Capital Humano". A continuación, se describen estos compromisos jurídicos.

La Constitución de la República de El Salvador de 1983 reconoce que: "Todas las personas son iguales ante la ley. Para el goce de los derechos civiles no podrán establecerse restricciones que se basen en diferencias de nacionalidad, raza, sexo o religión" (Art.3). "el derecho a la educación y a la cultura es inherente a la persona humana; en consecuencia, es obligación y finalidad primordial del Estado su conservación, fomento y difusión. El Estado propiciará la investigación y el quehacer científico" (Art. 53).

La Ley General de Educación de 2011 determina los objetivos generales de la educación, aplicada a todos los niveles y modalidades. Regula la prestación del servicio de las instituciones oficiales como privadas y establece como fines del sistema educativo: a) lograr el desarrollo integral de la personalidad en su dimensión espiritual, moral y social; b) inculcar el respeto a los Derechos Humanos y la observancia de los correspondientes deberes y c) combatir todo espíritu de intolerancia y de odio. Es importante destacar como un gran avance en materia de igualdad de género, en el ámbito de la educación, la reforma a este cuerpo legal en el artículo 5 - literal A -, el cual establece que el Ministerio de Educación deberá impulsar dentro del Sistema Educativo Nacional una Política de Equidad de Género, que incluya medidas específicas con el objetivo de eliminar los obstáculos que dificultan la igualdad objetiva entre los alumnos y las alumnas. Según este artículo, no se admitirá en los centros educativos del país las desigualdades entre los alumnos y las alumnas sustentadas en prejuicios o prácticas discriminatorias, basadas en una distribución estereotipada de papeles entre los sexos, y se garantizará en los centros educativos el uso de lenguaje no sexista. De igual forma, el artículo 76, literal A prohíbe que los centros educativos adopten medidas que impidan, limiten o perturben el inicio o continuidad normal de los estudios de las alumnas embarazadas o durante su período de lactancia, estableciendo que las autoridades de los centros deberán determinar, según el caso, las medidas de apoyo necesarias para garantizar la continuidad de los estudios de las alumnas embarazadas; la institución educativa que no dé cumplimiento a esta disposición incurre en una falta muy grave.

Ley de la Carrera Docente de 1996. Tiene como finalidad garantizar que la docencia sea ejercida por educadores registrados en el Ministerio, asegurándoles su estabilidad laboral para lograr una educación de calidad; de igual forma, establece derechos y obligaciones para los profesores y describe la organización administrativa que regula a los educadores. La ley establece la obligatoriedad de la denuncia y la sanción a los educadores que incurran en actos que afecten la integridad física y moral de los discentes, respetando la integridad física y moral de alumnos, alumnas o demás miembros de su comunidad educativa y denunciando cualquier hecho de violencia sexual dentro de la comunidad educativa. Teniendo en cuenta dichas obligaciones, se consideran faltas muy graves hacia estudiantes: aplicar cualquier forma de maltrato físico o psíquico que atente contra su dignidad, su integridad personal o el desarrollo de su personalidad; así como 
también acosar sexualmente o cometer actos contra la libertad sexual de compañeros o compañeras de trabajo, alumnos o alumnas, padres o madres de estos, dentro o fuera del centro educativo, lo cual trae aparejada una sanción de suspensión del cargo mientras se comprueban los hechos.

Reglamento de la Ley de Educación Superior de 2009. Establece en el Art. 11, las normas para el examen de los planes y programas de estudio de las Instituciones de Educación Superior, según las cuales la responsabilidad de examinar los programas es de la Dirección Nacional de Educación Superior, respetando la libertad de cátedra establecida para las Universidades, así como la calidad de la institución acreditada. Al respecto, no se autorizarán a las Universidades nuevos planes y programas de estudios, ni se autorizará la actualización de los planes y programas de estudio previamente aprobados, si del examen pertinente resulta que no cuenta con los requisitos mínimos para llevarlos a cabo con la calidad de la educación superior que se espera. Asimismo, fortalecerán dichos programas con contenidos relativos a: a) idioma español, b) idioma inglés, c) informática, d) educación ambiental, e) derechos humanos, f) educación inclusiva, g) gestión para la reducción del riesgo a desastres y $\mathrm{h}$ ) prevención a la violencia intrafamiliar y de género.

Ley de Protección Integral de la Niñez y Adolescencia, vigente desde el 2009. Constituye el cuerpo normativo más importante que garantiza el ejercicio de los derechos humanos. Establece que el Estado salvadoreño difundirá y promoverá el respeto a los derechos de toda niña, niño y adolescente y los derechos humanos en general, así como garantizar modalidades y horarios escolares especiales que permitan a adolescentes trabajadores asistir regularmente a sus centros de estudios; incluir en los programas educativos temas relacionados con la nutrición, la educación sexual y reproductiva, el embarazo precoz, la equidad y violencia de género, las drogas, las enfermedades infecto contagiosas y el medio ambiente y garantizar la permanencia en el ámbito escolar y no la discriminación de las niñas y las adolescentes madres, las embarazadas o las víctimas de violencia.

Ley Especial Integral para una Vida Libre de Violencia para las Mujeres, vigente desde el 2012. Tiene como propósito garantizar a las mujeres su derecho a una vida libre de violencia, comprendiendo este derecho el ser libres de toda forma de discriminación, ser valoradas y educadas libres de patrones estereotipados de comportamiento, prácticas sociales y culturales basadas en conceptos de sumisión o subordinación. Con relación al Ministerio de Educación, establece que a través de los programas y procesos educativos de enseñanza incluirá la promoción del derecho de las mujeres a vivir libres de violencia y discriminación, al igual que la generalización de las medidas destinadas a la prevención y erradicación de cualquier tipo de violencia contra las mujeres. Deberá eliminar de todos los programas educativos las normas, reglamentos y materiales que promuevan, directa o indirectamente, cualquiera de las formas de violencia contra las mujeres.

Las personas que ejerzan la dirección de los centros educativos públicos y privados adoptarán medidas para la detección y atención de los actos de violencia contra las mujeres, dentro del ámbito escolar. En las instituciones de educación superior se reglamentarán internamente acciones de detección y prevención de toda forma de violencia contra la mujer, al igual que impulsarán el desarrollo de investigaciones para la erradicación de la violencia contra las mujeres y el fomento de las relaciones de igualdad y no discriminación.

Ley de Igualdad, Equidad y Erradicación de la Discriminación contra las Mujeres, vigente desde el 2011. Ratifica el compromiso de la plena aplicación del principio constitucional de Igualdad de las personas y el cumplimiento de las obligaciones derivadas en este principio aplicables a la legislación nacional y a la acción efectiva de las instituciones del Estado. Tiene por objeto crear las bases 
jurídicas explícitas que orientarán el diseño y ejecución de políticas públicas para garantizar la igualdad real y efectiva entre mujeres y hombres, sin ningún tipo de discriminación.

El proyecto Capital Humano, implementado por el Ministerio de Educación (MINED) en el periodo 2016-2020, comprende dos grandes subgrupos de actividades:

\subsection{EL FORTALECIMIENTO DE LA CALIDAD EN LA EDUCACIÓN}

El MINED se encuentra incurso desde 2009 en la implementación del modelo de escuela inclusiva de tiempo pleno, favoreciendo el aumento de la jornada escolar desde 25 a 40 horas por semana, concentrando los recursos entre séptimo grado de primaria y bachillerato. La mejora curricular se centra en inglés, matemáticas, ciencias y tecnologías de la información. Bajo este enfoque, el modelo se está implementando a la fecha en 1.700 centros escolares de todo el país, conformando entre ellos 141 sistemas integrados que trabajan en la consolidación de todos los componentes del modelo. De ese universo de centros educativos, 378 son implementados a través de fondos del préstamo de Banco Mundial, 60 a través de la Cooperación Italiana - Universidad de Bolonia EducAid, 410 a través de USAID - FEDISAL y 506 escuelas están siendo asistidas con el esfuerzo propio de las direcciones departamentales del MINED. La Corporación Reto del Milenio (MCC) financia el programa en 349 escuelas agrupadas de 45 núcleos poblacionales, con 175.000 estudiantes.

Uno de los objetivos principales del proyecto "Capital Humano" es la transversalización del enfoque de género en el sistema educativo. Al menos 70.000 estudiantes de primaria hasta bachillerato serán formados desde el curso 18-19, en diversas áreas contempladas en la implementación de la Política de Equidad e Igualdad de Género del Ministerio de Educación (MINED), para lo cual el MINED cuenta, además de la financiación de MCC, con la colaboración de ONU Mujeres y la Universidad Centroamericana «José Simeón Cañas» (UCA).

La colaboración se está desarrollando desde febrero de 2018, y tiene previsto durar hasta abril de 2020, período en el cual se están implementando planes de igualdad de género y prevención de la violencia de género en los centros educativos, con la mejora los mecanismos de respuesta del MINED y de los centros educativos para la prevención, detección, registro y derivación de casos de violencias de género que ocurren en el ámbito educativo.

En Julio de 2018 ha comenzado la especialización de 300 docentes a nivel nacional en el curso de Posgrado "Especialización en educación no sexista y desarrollo humano", de 576 horas de formación certificada por el MINED y la UCA. Los módulos de formación incluyen educación incluyente no sexista, la formulación de planes para la equidad de género y la prevención de las violencias de género.

La actuación se concentra en los 45 núcleos poblacionales, que abarcan 349 centros educativos que apoya la Corporación Reto del Milenio (MCC) bajo el modelo de Escuela Inclusiva de Tiempo Pleno (SIEITP).

Asimismo, los estudiantes serán formados en áreas de prevención de violencia de género, fomento de relaciones de equidad en el ámbito escolar, libres de cualquier tipo de discriminación sexual. Además, se les dará formación en estrategias de empoderamiento social y prevención del embarazo en adolescentes, este último como factor que incide en la falta de educación de las niñas y adolescentes, entre otros. 


\subsection{LA REFORMA DEL SISTEMA DE EDUCACIÓN Y CAPACITACIÓN TÉCNICA VOCACIONAL (TVET).}

A través de esta actividad del MINED, dentro del proyecto "Capital Humano", con la asistencia técnica y financiera de la Corporación Reto del Milenio (MCC), se busca adecuar la capacitación de la fuerza laboral a las demandas del mercado.

Considerando que la educación es un instrumento clave para transformar estereotipos y roles culturalmente asignados a mujeres y hombres, que limitan significativamente las posibilidades y opciones de desarrollo personal de unas y otros, particularmente de las mujeres, es de vital importancia invertir en procesos educativos inclusivos y que incorporen los aspectos de género. Numerosos estudios demuestran que educar a las niñas genera importantes beneficios individuales y colectivos, a la vez que tiene un efecto positivo en el crecimiento económico. Estudios del Banco Mundial indican que por cada año de educación secundaria, aumenta un 18\% en el futuro el poder adquisitivo de la niña. (Banco Mundial, 2012). El acceso a la educación primaria es solo un primer paso para avanzar en la educación de las niñas. Adicionalmente se debe avanzar en su empoderamiento y su acceso a la igualdad efectiva de derechos, asegurar que las niñas aprenden en entornos seguros, que acceden y disfrutan de una educación de calidad, tanto secundaria como universitaria, y que acceden a áreas científico-técnicas tradicionalmente masculinas. Todas estas acciones son necesarias para que los beneficios de su educación alcancen su mayor impacto y sean sostenibles.

Una de las iniciativas más recientes y exitosas de capacitación técnico-profesional con perspectiva de género es la Ciudad de la Niñez y Adolescencia (CNA) de Santa Ana. Desde su inauguración, en el año 2016, la CNA ha tenido una inversión de 2.5 millones de dólares provenientes de la cooperación de la República de Taiwán y de fondos del Gobierno. Se han atendido a 29.547 niñas, niños y adolescentes, quienes han recibido atención en 12 talleres vocacionales y 6 programas de formación para la vida. La primera promoción de estos talleres ha graduado a 575 estudiantes de 13 centros escolares del departamento de Santa Ana, formando, con perspectiva de género, en talleres de informática, cosmetología, electricidad, arte en madera, electrónica, automotriz, estructuras metálicas, corte y confección, confección textil y robótica.

\section{CONCLUSIONES. PELIGROS CRÍTICOS EN IGUALDAD DE GÉNERO EDUCACIÓN}

El camino emprendido por la sociedad salvadoreña para conseguir la igualdad de género en la educación no universitaria es innegable. La implementación de numerosas leyes, la ayuda técnica de numerosos organismos, la colaboración de instituciones internacionales, y el apoyo financiero de las mismas permiten decir, con apoyo en los datos extraídos de los censos oficiales, que El Salvador está en la vanguardia de los países hispanoamericanos en las políticas de igualdad en el ámbito de la educación. Sin embargo, para no caer en triunfalismos, desglosaré en este apartado de conclusiones los riesgos que se presentan, después del 2020, en la igualdad de género en el ámbito educativo, es decir, los aspectos que dificultan la puesta en práctica de las políticas de igualdad de género.

\subsection{CONCLUSIÓN $1^{\text {a }}$}

Riesgo de no implementar la política de Equidad e Igualdad de género en el MINED y sus mecanismos de actuación más allá del 2020. 
En los Art. 10, 11 y 13 del Capítulo II de la Ley de Igualdad, Equidad y Erradicación de la Discriminación contra las Mujeres, LIE (2011), se establece que las instituciones del Estado deberán integrar los principios de igualdad y erradicación de la discriminación en todas las políticas, normativas, procedimientos y acciones desarrolladas en el ejercicio de sus competencias, así como instalar Unidades de Género y presupuestos de género institucionales. La Corporación Reto del Milenio (MCC) apoyó directamente al Ministerio de Educación en la formulación de la Política de Equidad e Igualdad de Género del MINED y su Plan de Implementación, que inició su ejecución en 2016.

Aun cuando el convenio MINED-MCC contempla el acompañamiento técnico y financiero para la ejecución del Plan de Implementación de la Política y para el MINED constituye una de sus prioridades, se hace necesario definir las estrategias de sostenibilidad en el tiempo de los compromisos adquiridos en esta Política. A este riesgo se suma que en el 2019 habrá elecciones presidenciales, situación que podría interrumpir la continuidad de la implementación del Plan, que tiene una duración prevista hasta 2020.

\subsection{CONCLUSIÓN $2^{\mathrm{a}}$}

La presencia de actitudes sexistas en los centros educativos y en las oficinas del MINED refuerza desigualdades de género.

En el país se ha investigado muy poco el sexismo en el ámbito educativo. El estudio de transversalización de género de FOMILENIO- MINED (2013) muestra la existencia de una tensión entre el discurso de igualdad de género, que empieza a instalarse en el ámbito educativo y los dispositivos de socialización de género que se reproducen en los centros educativos. Este resultado coincide con lo señalado por organizaciones internacionales sobre el hecho que en los centros escolares existen normas de género que socializan estereotipos sexuales y prácticas educativas que colocan a las niñas, adolescentes y mujeres en riesgo de vivir en condiciones de desigualdad y subordinación respecto a los hombres.

Las niñas y los niños tienden a ser educados de forma no sólo diferente sino antagónica y excluyente sobre la base de la creencia sexista sobre lo considerado "propio" del ser una mujer "femenina" en contraposición a lo considerado "propio" de un hombre "masculino", lo que presupone de fondo la superioridad del sexo masculino sobre el femenino, condición que limita las potencialidades de desarrollo de las niñas, así como de los niños en el presente y en el futuro con mayores desventajas sociales para las mujeres.

En las consultas realizadas en el marco de formulación de la política de género del MINED en el 2016, grupos focales de estudiantes adolescentes con una opción sexual diferente a la heterosexualidad, reconocen las limitaciones para acceder, mantenerse y egresar del sistema educativo regular debido a las marcadas situaciones de discriminación y violencia de las son que objeto en los centros educativos. Estas condiciones muestran la importancia de formular y desarrollar planes de formación dirigidos al personal docente, personal técnico- pedagógico del MINED y demás personas claves de forma que les faciliten proceso de toma de autoconciencia de la presencia de sesgos de género y la necesidad de la incorporación del enfoque de la igualdad de género e inclusión social en la cotidianidad escolar.

\subsection{CONCLUSIÓN $3^{\mathrm{a}}$}


Predominio de una división sexual de las áreas de estudio de educación media y de formación técnica y profesional.

Con relación a la segmentación por sexo existente en las áreas de estudio en formación técnica y educación superior, se identifica que las jóvenes se concentran en menos opciones técnicas y por lo general tienden mayoritariamente a estudios relativos a las áreas sociales y del cuidado (salud, educación, servicios). Por su parte, los jóvenes acceden a más y diversas opciones asociadas mayoritariamente a ámbitos técnicos. El problema de esta segmentación es que determina las opciones posteriores de unas y otros para insertarse en trabajos de mayor mercado competitivo y refuerza la brecha de ingresos entre mujeres y hombres a futuro.

El estudio de FOMILENIO-MINED (2013) señala que la persistencia de la segmentación en las opciones de estudio técnico-superior evidencia que el sistema educativo está reproduciendo patrones sociales y de desigualdad de género. Esta segmentación y elección de áreas de estudio son determinadas por las condiciones sociales y culturales que imponen ideas a niñas y niños sobre las áreas en que deberían desempeñarse. Por ello, el proyecto "Capital Humano" de los SIEITP puede contribuir a reducir las brechas a favor de mayor igualdad social y entre mujeres y hombres.

El problema no es tanto que mujeres y hombres estudien cosas distintas, sino que esas distintas elecciones generan después limitaciones y oportunidades diferentes para unas y otros para insertarse y progresar laboralmente, particularmente en empleos dignos y con buenas remuneraciones, y penalizando en mayor medida a las mujeres. Si bien estas elecciones resultan de una formación socio-cultural previa que perjudica el interés en asuntos técnicos en la población femenina, la escuela tiene el reto de contribuir a cambiar paradigmas y patrones tradicionales que orientan las elecciones de las adolescentes, de forma de incidir para que las adolescentes incursionen en áreas nuevas que respondan a las demandas del mercado laboral, limitando además el impacto en el crecimiento económico y el desarrollo nacional.

En el marco de la intervención en los centros educativos de los Sistemas Integrados de la Escuelas Inclusivas de Tiempo Pleno (SIEITP), mediante la creación de nuevas especialidades de bachillerato técnico, con el objetivo de potenciar mayores oportunidades de desarrollo laboral, como parte de la transversalización de género, se prevén acciones para favorecer una mayor equidad entre mujeres y hombres estudiantes en estas áreas, contribuyendo a erradicar la división sexual de los estudios.

En cuanto a las intervenciones vinculadas a la Formación Técnica y Profesional (FTP), de igual forma las principales oportunidades se orientan a lograr un cambio significativo en la actual segmentación por sexo en la educación y la formación técnica-profesional, y en la posterior inserción laboral de las mujeres en el mercado laboral. Se busca contribuir a establecer un sistema de formación técnica y profesional moderno que potencie las capacidades de mujeres y hombres para responder a las demandas del mercado y, por tanto, amplificar los impactos positivos del proyecto y su sostenibilidad a medio y largo plazo.

\subsection{CONCLUSIÓN $4^{\mathrm{a}}$}

Riesgos de género-sociales que afectan al acceso y la culminación de los estudios de tercer ciclo y bachillerato.

En El Salvador, la problemática de la culminación de los estudios está relacionada con factores como el estatus socio-económico, el nivel educativo de la madre, la insuficiente oferta educativa y la calidad educativa, entre otros factores, en algunos de los cuales puede incidirse en el marco de 
este proyecto. Al respecto, para el año 2014 (ENS, 2014) de todas las niñas y los niños que empiezan el primer grado, el 86 por ciento pasa al sexto grado. Al comparar por sexo se observa una leve diferencia que desfavorece a los niños, dado que el 88 por ciento de las niñas llega al sexto grado, frente al 84 por ciento de los niños (ENS, 2014).

Esta misma fuente muestra que el 96 por ciento de niños y niñas en los hogares del quintil más rico culminan la primaria comparado con el 77 por ciento de los hogares del quintil más pobre. A lo que se suma patrones culturales en las familias sobre la poca importancia de la educación frente al mundo del trabajo remunerado y al trabajo doméstico que se asigna a niños y niñas respectivamente.

Por otra parte, a menor nivel educativo de la madre es menor la proporción de niñas y niños que culminan la primaria y por ende su entrada al tercer ciclo. Así, el 67 por ciento de niños y niñas finaliza el sexto grado si la madre no tiene ningún nivel educativo, este porcentaje aumenta al 100 por ciento si la madre tiene un grado universitario. Esto refleja la importancia de impulsar acciones para elevar el nivel educativo de las niñas, adolescentes y jóvenes para que puedan ingresar y culminar el tercer ciclo y la educación media.

En cuanto a la oferta educativa, se estima en 454.349 la cifra de personas de entre 16 y 18 años que se encuentran en edad para asistir al nivel de educación media para el 2013 frente a la oferta de 890 centros educativos que prestan ese servicio, lo que significa un promedio de 510 estudiantes por centro educativo, una cantidad imposible de atender (EHPM, 2013, en UNICEF, 2014). MMC apoyará al MINED, dentro del proyecto "Capital Humano" para ampliar la oferta educativa en los 45 SIEITP en el nivel de educación media, factor que puede contribuir a aumentar la cobertura de grupos en condiciones de vulnerabilidad socio-económica y de género.

También existe el reto de contribuir a reducir aquellos riesgos sociales y de género relacionados con la calidad educativa en sí, mediante el conjunto de actividades educativas a desarrollarse en el marco de los SIEITP del proyecto "Capital Humano", entre estas, en los instrumentos curriculares, la formación docente, los sistemas de información, en las diferentes actividades de aprendizaje relativas al inglés, las TIC y habilidades para la vida y el trabajo, así como en la infraestructura escolar, para convertir los espacios educativos en ambientes amigables y que contribuyan a generar condiciones de seguridad para la integridad personal, tanto de niñas como de niños (mejor acceso a agua, servicios sanitarios separados por sexo y grupos de edad, medidas para la movilidad).

\subsection{CONCLUSIÓN 5a}

Alta proporción de embarazos y abandono escolar de niñas y adolescentes.

En el país, de acuerdo con la Encuesta Nacional de Salud (ENS, 2014), la tasa de fecundidad específica entre las adolescentes entre 15 y 19 de edad es de 74 nacimientos por cada 1.000 mujeres (66 para el área urbana y 87 para el área rural) y alcanza un pico de 122 nacimientos por cada 1.000 mujeres cuando tienen entre 20 y 24 años. Esta cifra disminuye posteriormente. La tasa de natalidad de adolescentes presenta una mayor proporción en la región oriental con 87 nacimientos por cada 1.000 mujeres.

De acuerdo con el MINSAL (2015) a nivel nacional, la proporción de partos de mujeres adolescentes entre el 2009 y 2014 no ha disminuido, sino que se mantiene en un promedio estimado de un poco más de 24.000 embarazos por año. Es decir, una de cada 5 adolescentes tuvo su primer parto antes de cumplir los 18 años (UNFPA, 2015) 
Los datos evidencian que a menor educación el indicador de la tasa de fecundidad en adolescentes aumenta, siendo 9 veces mayor en aquellas que poseen educación primaria comparada con mujeres con educación universitaria (ENS, 2014).

En los 45 municipios de los 8 departamentos donde se intervino con los SIEITP en 2015, la tasa de fecundidad de adolescentes entre 10 y 19 años fue del 42\%o. Es decir, por cada mil adolescentes, 42 adolescentes fueron asistidas en control prenatal por Ministerio de Salud en el año 2015.

A lo que se une la reconocida tendencia de abandonar los estudios por motivos de embarazo o hijos. El Censo de Matrícula Escolar Final del MINED (2015) proporciona información sobre el número de estudiantes que abandonaron por motivos de embarazo: 1164 en 2012; 1680 en 103; 1235 en 2014 y 1256 en 2015.

La problemática del embarazo en adolescentes se encuentra asociada a factores de orden social, económico, educativos y culturales. En lo que corresponde a educación queda evidenciada la necesidad de desarrollar acciones de educación y prevención de las uniones matrimoniales y del embarazo en niñas y adolescentes, y de la poca prioridad a la educación de las niñas en el ámbito de los SIEITP.

\subsection{CONCLUSIÓN 6a}

Altos niveles de violencia de género y sexual en los centros educativos, así como la falta de mecanismos de detección y seguimiento administrativo en el sistema educativo.

El Observatorio de Centros Educativos del Ministerio de Educación (2015) revela algunos datos sobre la problemática de la violencia sexual en los centros educativos del ámbito nacional, que constituyen la punta del iceberg de lo que puede ser esta problemática, dado que por naturaleza tiende a ser poco visibilizada: de un total de 5.132 centros educativos encuestados, 123 informan casos de violencia sexual entre estudiantes, 16 centros casos de violencia sexual cometida por docentes contra estudiantes y en 50 centros informan de denuncias por acoso sexual. Además, en 134 centros educativos reportaron la violación sexual como motivo de abandono.

La situación de la violencia sexual en el ámbito de los 45 municipios donde interviene "Capital Humano" con los SIEITP, se caracteriza por altos niveles de agresiones sexuales contra las niñas y adolescentes de 10 a 19 años. Para el año 2015 el Instituto Medicina legal reportó una tasa de agresión sexual del 18.46 por cada 10.000 niñas y adolescentes (IML, 2016).

Asimismo, los resultados de la Consulta Nacional con población LGTBI (SIS, 2012) revela que en los centros de estudio el $45.6 \%$ de entrevistados manifestó sufrir acoso y discriminación. Unido al dato que cuatro de cada 10 personas LGTBI de la muestra informan haber abandonado sus estudios por motivos de discriminación en los centros educativos. Sin duda, el reto parte de lograr una mayor comprensión de la condición y los derechos de la población LGTBI por parte del personal docente, adoptar mecanismos institucionales para mejorar la detección y sanción en todos los niveles y espacios educativos.

El sector educativo también juega un papel fundamental en el trabajo de prevención y erradicación de la violencia, no solo para que deje de ocurrir en el interior de los centros educativos, sino en su rol de formación y construcción del ejercicio de una ciudadanía respetuosa del derecho al ejercicio de la integridad personal y una vida libre de violencias. El sistema educativo, desde la educación 
preescolar hasta la educación superior, debe generar acciones de prevención y educación para la erradicación de la cultura de la violencia por razones de género u otra condición social. 


\section{BIBLIOGRAFÍA}

Banco Mundial (2012). Una (R)evolución de género en marcha: ampliación de las oportunidades económicas para las mujeres en América Central. Recuperado el 27 de julio de 2018 de: http://documentos.bancomundial.org/curated/es/901171468012925649/Una-R-evoluci243-n-de-g-233-nero-en-marcha-Ampliaci-243-n-de-las-oportunidades-econ-243-micaspara-las-mujeres-en-Am-233-rica-Central-Revisi-243-n-de-la-250-ltima-d-233-cada

Consejo por la Igualdad y la Equidad (CIE). Paso a paso hacia la igualdad de género en El Salvador: Tres lineas para la acción. San Salvador: 2009.

Departamento de Estado USA (2014). Reporte sobre trata de personas. Recuperado el 27 de julio de 2018 de: https://sv.usembassy.gov/es/our-relationship-es/official-reports-es/traffickingin-persons-report-2014-es/

DIGESTYC. Dirección nacional de estadísticas y censos (2013). Encuesta de Hogares de Propósitos Múltiples 2013. Recuperado el 27 de julio de 2018 de: http://www.digestyc.gob.sv/index.php/temas/des/ehpm/publicaciones-ehpm.html

DYGESTIC. Dirección nacional de estadísticas y censos (2014). Encuesta de Hogares de Propósitos Múltiples 2014. Recuperado el 27 de julio de 2018 de: http://www.digestyc.gob.sv/index.php/temas/des/ehpm/publicaciones-ehpm.html

FOMILENIO II/MINED (2013). Estudio para la transversalización de género en el sistema educativo. San Salvador: Inédito.

FUNDAUNGO. Fundación Guillermo Manuel Ungo (2016). Evolución de los homicidios en El Salvador, 2009-junio 2016, en Aportes al debate sobre la seguridad ciudadana, Núm 4. San Salvador. Recuperado el 27 de julio de 2018 de: http:/ / www.fundaungo.org.sv/products/evolucion-de-los-homicidios-en-el-salvador2009-junio-2016/41

García, P. (2005). Género, Educación y Política Pública, en La Ventana, Núm. 21. México

IML. Instituto de Medicina Legal (2016). Anuario estadístico 2015. San Salvador.

ISDEMU. Instituto Salvadoreño para el desarrollo de las mujeres (2013). Plan Nacional de Igualdady Equidad para las Mujeres Salvadoreñas. Recuperado el 27 de julio de 2018 de: http:/ /www.isdemu.gob.sv/index.php?option=com_phocadownload\&view=category\&id $=193 \% 3$ Aplan-nacional-de-igualdad-y-equidad-para-las-mujeressalvadoreas\&Itemid $=0 \&$ lang $=\mathrm{es}$

MINED (2014). Educación de El Salvador en cifras 2009-2013. Recuperado el 27 de julio de 2018 de: http://www.mined.gob.sv/index.php/estadisticas-educativas

MINED (2015). Censo de Matrícula Escolar. Recuperado el 27 de julio de 2018 de: http://www.mined.gob.sv/index.php/estadisticas-educativas 
MINSAL. Ministerio de Salud Pública y Asistencia Social (2014). Encuesta nacional de Salud. Recuperado el 27 de julio de 2018 de: https://www.unicef.org/elsalvador/ENS-Finalweb.compressed.pdf

MINSAL. Ministerio de Salud Pública y Asistencia Social (2015). Memoria de labores. Recuperado el 27 de julio de 2018 de: http://www.salud.gob.sv/memoria-de-labores-2015-2016/

OCDE (2014). Cerrando las brechas de género: es hora de actuar. Recuperado el 27 de julio de 2018 de: https://www.oecd-ilibrary.org/social-issues-migration-health/cerrando-las-brechas-degenero_9789264208582-es

OEA. Organización de Estados Americanos (2016).Observatorio de seguridad ciudadana. Recuperado el 27 de julio de 2018 de: http://www.oas.org/dsp/observatorio/database/countriesdetails.aspx?lang=es\&country $=\mathrm{SLV}$

PNUD. Programa de Las Naciones Unidas para el Desarrollo (2011). Informe sobre Desarrollo Humano 2011. Sostenibilidad y equidad: Un mejor futuro para todos. Recuperado el 27 de julio de 2018 de: http://www.sv.undp.org/content/el_salvador/es/home/library/hiv_aids/informesobre-desarrollo-humano-2011.html

Ruiz-Jarabo, C. y Blanco Prieto, P. (2005). La violencia contra las mujeres: Prevención y detección. Madrid: Díaz de Santos.

SIS. Secretaría de Inclusión Social (2012). Consulta Nacional sobre realidades LGBTI en El Salvador. San Salvador: Secretaría de Inclusión Social.

UNFPA. FONDO DE POBLACIÓN DE LA O.N.U (2016). Mapa de embarazos en niñas y adolescentes en El Salvador. Recuperado el 27 de julio de 2018 de: http://www.inclusionsocial.gob.sv/wp-content/uploads/2016/09/El-Salvador-Mapa-deEmbarazos-2015.pdf

UNICEF (2014). Informe de la Situación de la niñezy adolescencia en el Salvador. Recuperado el 27 de julio de 2018 de: https://www.unicef.org/elsalvador/Informe_de_situacion_de_la_NNA_en_El_Salvador .pdf 


\section{SOBRE EL AUTOR}

\section{Carlos Martínez Herrer}

Doctor en Ciencias de la Educación por la UCV. Profesor doctor agregado de la U. Católica de Valencia.

Licenciado en Derecho por la Universidad de Valencia. Funcionario del Ministerio de Justicia, en excedencia

Profesor Practicum y Tercer Ciclo en U. de Valencia. Jefe de departamento didáctico en IES de la Conselleria de Educación GVA.

Publicaciones sobre: Historia de la Educación, Formación y Orientación Laboral, Nuevas Tecnología en la Educación, Educación Católica.

Línea de investigación en Historia de la Educación Católica. La presencia jesuita en la Historia reciente de la ciudad de Valencia.

Contact information: carlos.martinez@ucv.es

Universidad Católica de Valencia. Facultad de Ciencias de la Educación. Despacho 16130. Calle Sagrado Corazón, 546110 Godella (Valencia) 